\title{
Q1 Spatial attention enhances object coding in local and distributed representations of the lateral occipital complex
}

\author{
22 Matthias Guggenmos a,b,*, Volker Thoma ${ }^{c}$, John-Dylan Haynes ${ }^{\mathrm{a}}$, Alan Richardson-Klavehn ${ }^{\mathrm{d}}$, \\ Radoslaw Martin Cichy ${ }^{\mathrm{e}, 1}$, Philipp Sterzer ${ }^{\mathrm{a}, \mathrm{b}, 1}$ \\ a Bernstein Center for Computational Neuroscience, Berlin, Germany \\ b Visual Perception Laboratory, Charité Universitätsmedizin, Berlin, Germany \\ c School of Psychology, University of East London, London, UK \\ d Department of Neurology, Otto von Guericke University, Magdeburg, Germany \\ e Computer Science and Artificial Intelligence Laboratory, Massachusetts Institute of Technology, Cambridge, USA
}

\section{A R T I C L E I N F O}

\section{Article history:}

Received 30 December 2014

Accepted 1 April 2015

Available online $\mathrm{xxxx}$

\section{Keywords:}

Attention

Objects

Lateral occipital complex

Multivariate pattern analysis

Mutual information

fMRI

\begin{abstract}
A B S T R A C T
The modulation of neural activity in visual cortex is thought to be a key mechanism of visual attention. The inves- 22 tigation of attentional modulation in high-level visual areas, however, is hampered by the lack of clear tuning or 23 contrast response functions. In the present functional magnetic resonance imaging study we therefore systemat- 24 ically assessed how small voxel-wise biases in object preference across hundreds of voxels in the lateral occipital 25 complex were affected when attention was directed to objects. We found that the strength of attentional modu- 26 lation depended on a voxel's object preference in the absence of attention, a pattern indicative of an amplificatory 27 mechanism. Our results show that such attentional modulation effectively increased the mutual information be- 28 tween voxel responses and object identity. Further, these local modulatory effects led to improved information- 29 based object readout at the level of multi-voxel activation patterns and to an increased reproducibility of these 30 patterns across repeated presentations. We conclude that attentional modulation enhances object coding in 31 local and distributed object representations of the lateral occipital complex.
\end{abstract}

(c) 2015 Published by Elsevier Inc.

\section{Introduction}

Attention is a cognitive process that enables us to focus on certain aspects of the environment for the benefit of improved performance (Bashinski and Bacharach, 1980; Cameron et al., 2002; Carrasco et al., 2000; Hawkins et al., 1990). One way in which attention has been found to impact neural processing is through an amplification of neural responses to attended spatial locations, objects, or features (for review, see Treue, 2003). In the visual domain, attentional amplification has been found throughout the visual processing hierarchy, from the earliest stage of visual neural processing in the lateral geniculate nucleus (O'Connor et al., 2002), primary visual cortex (Gandhi et al., 1999; Martínez et al., 1999; Somers et al., 1999), up to high-level visual cortices (Murray and Wojciulik, 2004; O'Craven et al., 1999; Serences et al., 2004) and the frontal lobes (Gitelman et al., 1999). However, the nature of attentional modulation remains a topic of debate. A number of studies have reported that attention leads to a multiplicative scaling of neuronal responses (Di Russo et al., 2001; McAdams and Maunsell, 1999; Treue

* Corresponding author at: Bernstein Center for Computational Neuroscience, Philippstraße 13, Haus 6, 10115 Berlin, Germany.

E-mail address: matthias.guggenmos@bccn-berlin.de (M. Guggenmos).

1 Contributed equally. and Martínez Trujillo, 1999; Treue and Maunsell, 1999), which results 55 in an increase of a neuron's signal to noise ratio. In contrast, other stud- 56 ies reported results that violated the predictions of the multiplication 57 hypothesis, by showing that spatial attention leads to increased neural 58 responses in visual areas in the absence of any visual stimulation 59 (Kastner et al., 1999; Luck et al., 1997; Ress et al., 2000; Silver et al., 60 2007). According to these studies, attentional modulation involves an 61 unspecific baseline shift of activity.

A common approach to investigate the effects of visual attention is 63 the recording of neural responses across a range of a stimulus parameter 64 (e.g., orientation of motion direction) both in the presence and absence 65 of attention. In this way, previous studies have examined the attentional 66 modulation of single-neuron (McAdams and Maunsell, 1999; Motter, 67 1993; Treue and Martínez Trujillo, 1999) or voxel (Saproo and 68 Serences, 2010, 2014) tuning profiles. However, a complicating factor 69 for the investigation of attentional modulation in high-level object- 70 coding areas like the human lateral occipital complex (LOC) is the lack 71 of analogous neuronal tuning functions. Similarly, the analysis of con- 72 trast response functions - a technique that has been used to study the 73 nature of attentional modulation for low-level visual stimuli 74 (Reynolds et al., 2000; Williford and Maunsell, 2006) - is problematic, 75 because object-related neuronal responses become increasingly invari- 76 ant to contrast along the visual hierarchy (Avidan et al., 2002; Rolls and 77 
Baylis, 1986) and this invariance may itself depend on attention (Murray and $\mathrm{He}, 2006$ ). In the present work we therefore used a different approach by exploiting the fact that the LOC represents objects in a distributed fashion across ensembles of neural populations (Haxby et al., 2001; Rice et al., 2014). At the spatial resolution of fMRI this distributed code is expressed in a differential preference of voxels for a given stimulus, likely representing the cumulative stimulus preference of neurons within these voxels. Thus, if attention causes an amplification of neural activity as opposed to a mere baseline shift, these preferences should be augmented with attention, and as a consequence single- and multi-voxel responses should become more informative about the stimuli encoded in these voxels.

In the present study we presented human participants with objects under conditions of spatial attention and inattention in a functional magnetic resonance imaging (fMRI) experiment. We had two aims. First, we sought to probe the nature of attentional modulation of visual object responses in the LOC as described above, by examining whether attentional modulation increased with a voxel's preference for a given object in the absence of attention, or whether the modulation was independent of object preference. In a second step we investigated whether these local modulatory effects of attention resulted in a more informative and reliable object code. To this end we used a mutual information metric (Saproo and Serences, 2010; Serences et al., 2009) to assess whether single-voxel responses became more informative about object identity with attention. At the multi-voxel pattern level we examined how these local changes affected the quality of object representations through pattern similarity and classification-based analyses.

\section{Materials and methods}

\subsection{Disclosure}

A previous article (Guggenmos et al., 2015) was based on the same fMRI dataset, but pursued a different research question and orthogonal analyses.

\subsection{Participants}

Eighteen healthy participants ( 11 female, mean age \pm SEM, $23.4 \pm 0.8$ years) took part in the experiment for payment after giving written informed consent. The study was conducted according to the declaration of Helsinki, and approved by the local ethics committee.

\subsection{Experimental design}

116

Our key experimental manipulation was to direct participants' spa- 117 tial attention to either an object (attended condition) or a noise stimu- 118 lus (unattended condition). Overall the experimental design comprised 119 the factors attention (attended, unattended) as a factor of interest, as 120 well as object (camera, watering can, chair), configuration (intact, 121 split) and side of presentation (left, right) as factors of no interest. Con- 122 figuration was manipulated by minimally scrambling (half-splitting) 123 the objects, but note that the analyses in this article were based on intact 124 objects only. Within each of 8 experimental runs, an object appeared in 125 4 trials in each attention condition (in 2 trials per side of presentation). 126 The order of presentation was randomized across the 48 trials of each 127 run.

\subsection{Experimental procedures}

129

In each trial (Fig. 1A), participants viewed a stimulus display that 130 contained an object and a noise stimulus on either side of a central 131 fixation cross. Spatial attention was manipulated by means of a 132 brightness discrimination task that was performed either on the ob- 133 ject (attended condition) or the contralateral noise stimulus (unat- 134 tended condition). A trial (Fig. 1A) started with a blank fixation 135 screen for $3300 \mathrm{~ms} \pm 2000 \mathrm{~ms}$, after which one half of a central 136 black fixation diamond turned red, indicating the side to which at- 137 tention should be directed. Following this cue and a short fixed inter- 138 val $(250 \mathrm{~ms})$, four repetitions of the stimulus-response phase 139 appeared. Each stimulus-response phase lasted $1500 \mathrm{~ms}$ and com- 140 prised the presentation of the stimulus screen $(500 \mathrm{~ms})$, a pattern 141 mask ( $133 \mathrm{~ms}$ ) and a response screen $(867 \mathrm{~ms})$. The object appeared 142 on one side of the fixation cross (offset $3.84^{\circ}$ of visual angle) and a 143 noise stimulus at the same offset on the other side of the stimulus 144 screen. All visual stimuli subtended 3.81 by $3.81^{\circ}$ of visual angle. A 145 brightness change occurred $283 \mathrm{~ms}$ after stimulus onset simulta- 146 neously on both the object and the noise stimulus, such that they be- 147 came independently and randomly either darker or lighter. 148 Participants were instructed to press a button on the response box 149 when the stimulus on the cued side became darker. Responses 150 were counted as valid within a time window of $1000 \mathrm{~ms}$ after stimu- 151 lus offset. In each repetition of the stimulus-response phase, the 152 same object was shown at the same position. The noise stimulus, 153 while also presented at the same position, was randomly generated 154 for each repetition.

155

To independently identify object-responsive regions of the lateral 156 occipital complex (LOC) in each participant (Malach et al., 1995), we Q4

\section{A}

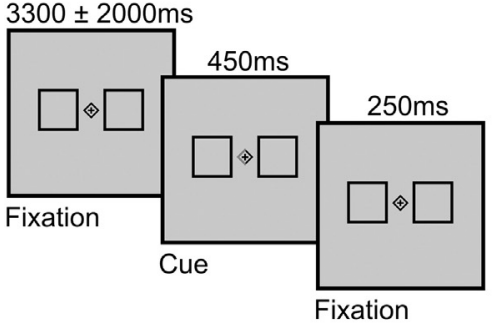

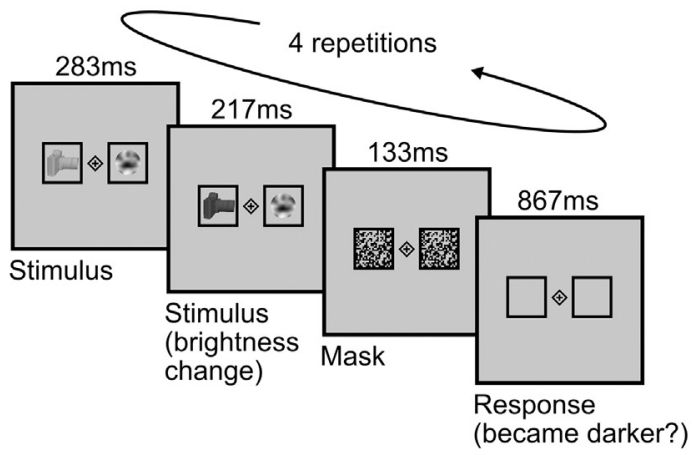

B

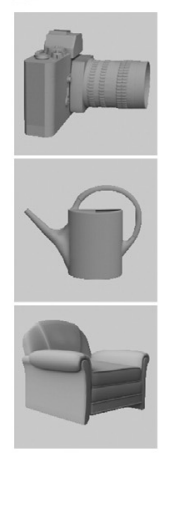

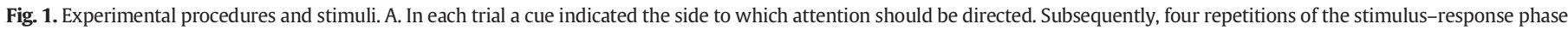

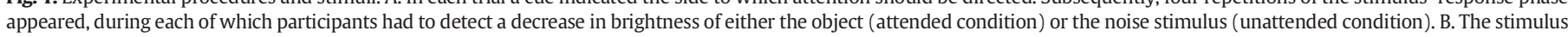
set consisted of three objects in an intact and half-split configuration. 
conducted a localizer run with 5 blocks of intact objects, 5 blocks of split objects and 10 blocks of grid-scrambled versions of the objects in randomized order. Blocks lasted for $15.8 \mathrm{~s}$ during which 20 images were presented for $600 \mathrm{~ms}$ each, followed by $200 \mathrm{~ms}$ blank screen. Pairs of identical objects were shown left and right of fixation, equaling the configuration of the main experiment in eccentricity and size. Participants performed a one-back task on the object pairs, in which they had to indicate via button press whenever the same stimulus display appeared twice in a row.

\subsection{Stimuli}

Stimuli were generated with Psychophysics Toolbox 3 (http:// psychtoolbox.org) and projected with a Sanyo LCD projector at $60 \mathrm{~Hz}$. The stimulus set consisted of three grayscale objects (camera, watering can, chair) based on realistic three-dimensional models presented either intact or half-split (Fig. 1B). The objects were selected for representing non-overlapping man-made categories to increase the discriminability of evoked neuronal activation patterns. The noise stimuli matched the objects in terms of spatial extent and complexity to ensure that there would be no performance difference. They were randomly generated for each trial by sampling a $9 \times 9$ random binary matrix, scaling the matrix to $216 \times 216$ pixels, applying a low-pass filter with a cutoff frequency of 0.02/pixel and cropping pixels outside a circle of 216 pixels diameter. This procedure resulted in circular grayscale stimuli with randomly distributed smooth patches that approximately matched the objects in terms of spatial extent. Both the objects and the noise stimuli were scaled to grayscale RGB values between 50 and 205. To generate these brightness changes, the underlying RGB histograms were shifted up or down by 50 (the image background remained constant with an RGB value of 200). The pattern masks were generated for each trial by sampling an $18 \times 18$ random binary matrix and scaling the matrix to $216 \times 216$ pixels.

\subsection{Eyetracking}

Eyetracking data were successfully collected in 16 of 18 subjects using an infrared video eyetracking system (iView XTM MRI $50 \mathrm{~Hz}$, SensoMotoric Instruments, Teltow, Germany). For each run, the horizontal eye movement data were low-pass filtered and drift corrections were performed. As a measure of fixation reliability, we computed the percentage of recorded eye gaze positions during stimulus presentation within a $1.93^{\circ}$ visual angle circle around the center of the fixation cross. This radius corresponded to the eccentricity of the inner edges of the two stimulus-containing boxes (see Fig. 1A). In addition, we computed the number of saccades to the intact objects and the noise stimuli, separately for the attended and the unattended condition. Saccades were defined as events of at least three consecutive data points in velocity space exceeding a velocity criterion of $30^{\circ} / \mathrm{s}$. Saccades were counted as object-directed or noise-directed saccades, when their endpoint was located within the object-containing box, or the noise-containing box, respectively.

\subsection{FMRI data acquisition and preprocessing}

FMRI data were acquired on a 3-Tesla Siemens Trio (Erlangen, Germany) scanner using a gradient echo planar imaging (EPI) sequence and a 12-channel head-coil. We recorded 8 experimental runs of 214 whole-brain volumes each, and one LOC localizer run of 242 volumes ( TR $=2 \mathrm{~s}$, echo time (TE) $25 \mathrm{~ms}$, flip angle $78^{\circ}, 33$ slices, $3 \mathrm{~mm}$ isotropic resolution, interslice gap $0.75 \mathrm{~mm}$ ). In addition, a high-resolution T1weighted image was acquired (TR $=1.9 \mathrm{~s}$, echo time (TE) $2.51 \mathrm{~ms}$, flip angle $9^{\circ}, 192$ slices, resolution $1 \mathrm{~mm}$ isotropic). The data of the experimental runs were realigned using SPM8 (Wellcome Department of Imaging Neuroscience, Institute of Neurology, London). Data analyses for the main experiment were generally performed in native subject space. An exception was an illustrative display of the whole-brain 218 group-level T-maps for the main effect of attention, for which we gener- 219 ated spatially normalized (MNI) and smoothed ( 8 mm Gaussian kernel) 220 volumes. Preprocessing of the localizer data included realignment, spa- 221 tial normalization to an MNI template and smoothing ( $8 \mathrm{~mm}$ Gaussian 222 kernel).

\subsection{FMRI data analysis}

2.8.1. First-level general linear models (GLMs)

For each participant we estimated a GLM including the stimulus- 226 onset regressors, accounting for the factors attention (attended, unat- 227 tended), object (camera, can, chair) and configuration (intact, split). 228 The onsets of each experimental regressor were set to the beginning 229 of the stimulus-response phase. In addition, six motion parameters 230 were included as regressors-of-no-interest. All experimental regressors 231 were modeled as stick functions and convolved with a canonical hemo- 232 dynamic response function.

The GLM for the functional localizer comprised regressors for objects 234 and scrambled objects and six motion parameters. The experimental re- 235 gressors were modeled as boxcar functions with durations equal to the 236 block lengths (15.8 s) and convolved with a canonical hemodynamic re- 237 sponse function as implemented in SPM8.

\subsubsection{Region of interest procedures}

Our region of interest (ROI) was the LOC, a functionally defined 240 region that responds more to images of objects than their counter- 241 parts and stretches from the lateral occipital cortex to posterior fusi- 242 form gyrus (Grill-Spector et al., 1999). We anatomically constrained Q5 the LOC by a bilateral composite mask of the inferior occipital cortex, 244 middle occipital cortex and the posterior half of the fusiform gyrus 245 (derived from the AAL Atlas, Tzourio-Mazoyer et al., 2002). Then Q6 the LOC ROI was defined as the intersection of the anatomical mask 247 and the functional localizer based on the t-contrast intact 248 objects $>$ scrambled objects of the functional localizer at a significance 249 level of $\mathrm{p}<0.05$ (family-wise error (FWE) corrected at the whole- 250 brain level). Note that this t-contrast was a group-level t-contrast, 251 because the statistical power in the first-level localizer contrasts 252 was not sufficient to define individual ROIs in all participants at the 253 $\mathrm{p}_{\mathrm{FWE}}<0.05$ level. To ensure a homogenous generation of the LOC 254 ROI for all participants we thus first defined the LOC ROI in group- 255 level (MNI) space and subsequently reverse-normalized the gener- 256 ated ROI to each participant's native space.

\subsubsection{Quantifying changes in mean BOLD activity}

To estimate neural activity in the LOC ROI and its dependence on at- 259 tention, we extracted the voxel-wise beta values for attended and unat- 260 tended objects separately and averaged across objects and voxels. This 261 procedure resulted in single values representing the average BOLD re- 262 sponse to attended and unattended objects.

To visualize the spatial extent of the attentional modulation at a 264 whole-brain level, we performed a group-level repeated-measures 265 ANOVA with factors attention and object and computed the post-hoc 266 contrast attended $>$ unattended. This analysis was based on normalized 267 and smoothed data. Voxels were considered statistically significant at 268 a level of $\mathrm{p}<0.05$, FWE-corrected at the whole-brain level.

2.8.4. Analyzing attentional modulation as a function of object preference 270

We next analyzed whether the attentional modulation depended on 271 the preference of a voxel for a given object. We reasoned that if atten- 272 tion leads to an amplification of neural responses, the difference be- 273 tween a voxel's attentional modulation for the preferred object and 274 the modulation for the non-preferred objects should increase as a func- 275 tion of object preference. By contrast, if attention led to an unspecific 276 baseline shift irrespective of a voxel's preference for the presented ob- 277 ject, the attentional modulation should not differ between the 278 
presentations of the voxel's preferred and non-preferred object. We therefore defined a preference index $\operatorname{PI}(i)$ for each object $i$ and each voxel based on the data of the unattended condition:

$\mathrm{PI}(i)=\beta_{\text {unatt }}(i)-\left\langle\beta_{\text {unatt }}(i)\right\rangle$,

where $\beta_{\text {unatt }}(i)$ and $\beta_{\text {unatt }}(\mid i)$ are the voxel-wise beta values in the unattended condition for object $i$ and all objects except $i$ (denoted as "not i": ii) respectively; the symbol $<>$ denotes the average operation (here across objects). PI was based on the unattended condition to circumvent the potential issue that the object preference of a voxel in the attended condition might not be independent of the magnitude of the attention effect. To compute the strength of the attentional modulation for an object $i$ relative to the other objects $\backslash i$, we defined a relative attentional modulation index $\operatorname{RAI}(i)$ as follows:

$\mathrm{RAI}(i)=\beta_{\mathrm{att}}(i)-\beta_{\mathrm{unatt}}(i)-\left\langle\beta_{\mathrm{att}}(i)-\beta_{\mathrm{unatt}}(i)\right\rangle$,

where $\beta_{\text {att }}(i)$ and $\beta_{\text {att }}(\mid i)$ are the voxel-wise beta values in the attended condition for object $i$ and all objects except $i$ respectively.

Finally, we quantified the RAI as a function of PI. To preclude a selection bias we used a leave-one-run-out procedure, such that PI and RAI were computed on independent data. The leave-one-run-out procedure was performed for each object $i$ separately as follows. In each fold, we sorted the pooled voxels from the LOC ROI according to PI $(i)$ based on data from all but one experimental runs. We then divided the sorted voxels into 10 equinumerous bins (deciles) according to $\mathrm{PI}(i)$ and computed the average $\operatorname{RAI}(i)$ for the voxels in each bin based on the data of the held-out run. Subsequently, we computed an average RAI across objects for each bin, resulting in a single RAI for each bin.

\subsubsection{Computing the mutual information between BOLD response and pre- sented objects}

To investigate whether attention increased object information encoded in the activity of individual voxels, we used a mutual information (MI) metric. MI estimates the extent to which the uncertainty about one variable $Y$ (here: BOLD response to the object being presented) is reduced by measuring another variable $X$ (here: the object being presented) (cf. Saproo and Serences, 2010; Serences et al., 2009). The mutual information (MI) measure is defined as the difference of the total entropy $H(X)$ and the noise entropy $H(X \mid Y)$ :

$$
\begin{aligned}
\operatorname{MI}(X ; Y) & =H(X)-H(X \mid Y) \\
& =-\sum_{x \in X} p(x) \log _{2} p(x)-\left(-\sum_{y \in Y} p(y) \sum_{x \in X} p(x \mid y) \log _{2} p(x \mid y)\right) .
\end{aligned}
$$

Thus we subtract from the total entropy $H(X)$, which corresponds to the overall dynamic range of responses, the noise entropy, which is a measure for the noise in the data conditional on each presented object. The remainder quantifies to what degree the variation in the BOLD signal is informative about the presented object. To compute the total and noise entropies, estimated BOLD responses were transformed into a discrete variable $(X)$ by dividing the entire range of responses into a set of 10 equinumerous bins (deciles). This discretization was based on the pooled range of responses from all voxels in either the attended or the unattended condition after subtracting out the respective mean activation levels of the attended and the unattended condition. This subtraction was done to avoid errors in the binning process due to additive shifts attributed to attention (Saproo and Serences, 2010). In the above formulation, $p(x)$ corresponds to the frequency with which a response in a given voxel falls into bin $x$. The noise entropy term $H(X \mid Y)$ additionally required the computation of the probability $p(y)$ of each object $y-1 / 3$ in our case, given that the experiment consisted of three equally often appearing objects - and $p(x \mid y)$, which corresponds to the frequency with which a response in a given voxel falls into bin $x$, given object $y$ was presented. We normalized the mutual information for each participant to a range between 0 and 1 by dividing $\operatorname{MI}(X ; Y) 334$ by the total entropy $H(X)$ (Kojadinovic, 2005). A normalized MI value 335 of 0 indicates that BOLD response $X$ and object label $Y$ are completely in- 336 dependent, whereas a normalized MI value of 1 indicates that response 337 $X$ gives complete information about the object label $Y$. The MI metric 338 was applied to the responses of attended and unattended objects 339 separately.

2.8.6. Analyzing the effects of attention at the multi-voxel pattern level 341

To assess the effect of attention at the multi-voxel pattern level, we 342 examined object-related activation patterns with and without attention 343 by means of a pattern similarity measure and support vector machine 344 (SVM) classification. The two methods are complementary in the 345 sense that the similarity measure provided a transparent quantification 346 of the reproducibility (within-object pattern similarity) across runs, 347 whereas the SVM classification assessed the amount of information 348 that can be read out from these activation patterns.

\subsubsection{Support vector machine classification}

350

Support vector machine classification (SVM) was performed using 351 The Decoding Toolbox (Hebart et al., 2014) with a linear C-SVM and a 352 fixed cost parameter $(c=1)$. We quantified object information in the 353 LOC for attended and unattended objects. We trained the classifier to 354 discriminate between objects based on multi-voxel activation patterns 355 in the LOC ROI in native subject space (Haynes and Rees, 2005; 356 Kamitani and Tong, 2005). A leave-one-run-out cross-validation proce- 357 dure was used, such that in each of 8 folds the classifier was trained on 358 the beta maps of seven runs and tested on the left out eighth run. We 359 performed pair-wise decoding between the three pairs of objects (cam- 360 era-can, camera-chair, can-chair) separately for the attended and the 361 unattended condition. Subsequently the decoding accuracies were aver- 362 aged across folds and object pairs.

\subsubsection{Pattern similarity analysis}

364

The pattern similarity analysis was based on z-transformed correla- 365 tions between activation patterns in the LOC ROI. The within-object pat- 366 tern similarity (WPS) measured the correlation between the patterns 367 evoked by the same object across the 8 runs (separately for attended 368 and unattended objects). For each object this led to $8 \cdot(8-1) / 2=369$ 28 correlation coefficients for the pairwise combinations of runs, 370 which were z-transformed and averaged across permutations and ob- 371 jects. This procedure yielded a single within-object pattern similarity 372 value for both the attended and the unattended condition. As a control 373 analysis, we also computed the between-object pattern similarity (BPS). 374 BPS was assessed analogously to WPS, except that the correlation coef- 375 ficients were computed between patterns evoked by different objects, 376 resulting in three between-object comparisons (camera-can, camera- 377 chair, can-chair). To avoid an overestimation of pattern similarity due 378 to within-run autocorrelations, we excluded all within-run compari- 379 sons (Mumford et al., 2014).

\section{Results}

\subsection{Behavioral results and fixation control}

Participants detected and reported brightness changes of the objects 383 and the noise stimuli highly accurately (performance $>98 \%$ ), indicating 384 focused attention on the correct stimulus. On average, $98.3 \pm 0.8 \% 385$ (mean \pm SEM) of recorded eye gaze positions during stimulus presenta- 386 tion were within the fixation area, demonstrating that the participants 387 maintained fixation throughout the experiment. There was no difference 388 in the overall number of saccades between the attended and the unat- 389 tended condition (attended: $3.1 \pm 1.6$ saccades in the experiment; unat- 390 tended: $3.6 \pm 2.2 ; \mathrm{p}=0.43, \mathrm{t}(15)=-0.80$, two-tailed t-test), and 391 neither was there a difference with respect to the number of object- 392 directed (attended: $2.9 \pm 1.6$; unattended: $0.9 \pm 0.6$; $\mathrm{p}=0.19,393$ 
$\mathrm{t}(15)=1.36$ ) or noise-directed saccades (attended: $0.2 \pm 0.2$; unattended: $2.8 \pm 2.0 ; \mathrm{p}=0.22, \mathrm{t}(15)=-1.26)$. The interaction of saccade direction (object-directed, noise-directed) and attention (attended, unattended) was not significant $(\mathrm{p}=0.21, \mathrm{~F}(1,15)=1.71$, repeatedmeasures ANOVA). These results, as well as the low absolute number of object- or noise-directed saccades, indicate that differences between the neural correlates of the attended and the unattended condition are unlikely to ensue from effects of eye movements.

\subsection{Attention amplifies responses to objects in the lateral occipital complex}

To examine the influence of covert attention on neural activity, we compared the overall average BOLD response for attended and unattended objects within the LOC averaged over objects and sides of presentation. Attended objects led to a significant increase of neural activation $(\mathrm{p}<0.001, \mathrm{t}(17)=5.00$, Cohen's $\mathrm{d}=1.17$; Fig. $2 \mathrm{~A})$.

In order to test whether the effect of the attention manipulation was confined to object-selective cortex, we quantified the overlap between the thresholded ( $\mathrm{p}_{\mathrm{FWE}}<0.05$ ) whole-brain T-maps of the contrasts attended $>$ unattended (main experiment) and intact $>$ scrambled (functional localizer). We found that $94.8 \%$ of the voxels showing an effect in the attention contrast overlapped with voxels classified as objectselective (Fig. 2B). Thus our focus on the LOC was justified by the spatial extent of the attentional modulation. It should be noted, however, that the attended and the unattended conditions differed only with respect to the attended stimulus type (object vs. noise pattern), but neither systematically with respect to low-level features (likely canceling out effects of attention in earlier visual areas in the contrast attended $>$ unattended) nor with respect to task (likely canceling out effects of attention in executive cortices). The spatial restriction of attentional modulation to LOC therefore reflects a deliberate property of our design, rather than the absence of attentional modulation in other brain areas.

\subsection{Attention modulates neural activity as a function of object preference}

We reasoned that if attention led to an amplification of neural activity (as opposed to a mere baseline shift), the attentional modulation should be greater for a voxel's preferred object relative to its nonpreferred objects. An analysis of a voxel's attentional modulation for a given object in dependence of its preference for the object should thus be informative about the specificity of the attentional modulation. To quantify the difference between the attentional modulation for preferred and non-preferred objects, we computed a relative attentional modulation index (RAI). Further, we determined a preference index (PI) for each voxel based on the mean response to a given object relative to the response of the other objects in the unattended condition. We hypothesized that RAI should increase as a function of PI.

To this end, we used a leave-one-run-out procedure, in which we sorted the voxels according to their PI, divided the voxels into 10 equinumerous bins (deciles) and computed the average RAI for each bin. We found that the RAI increased as a function of PI (linear slope 440 $[$ mean \pm SEM]: $0.066 \pm 0.022, \mathrm{p}=0.009, \mathrm{t}(17)=3.00$, two-tailed $\mathrm{t}-441$ test against the null hypothesis of a slope of zero; Fig. 3). In direct com- 442 parison, the average RAI for preferred objects ( $\mathrm{PI}>0$ [mean $\pm \mathrm{SEM}$ ]: 443 $0.18 \pm 0.06)$ was greater than the average RAI for non-preferred objects 444 $(\mathrm{PI}<0:-0.17 \pm 0.06)(\mathrm{p}=0.011, \mathrm{t}(17)=2.86$, Cohen's $\mathrm{d}=0.67) .445$ These results show that the modulation of neural activity through spa- 446 tial attention comprises an amplificatory component and is not due to 447 a baseline shift only.

3.4. Attention increases the mutual information between BOLD responses 449 and object identity

To test whether the increase of neural activity increased a voxel's in- 451 formation about the presented objects, we computed the mutual infor- 452 mation for attended and unattended objects. We found that attention 453 increased the mutual information of a voxel's responses about the ob- 454 jects presented $(p<0.001, t(17)=5.72$, Cohen's $d=1.35)$. The per- 455 centage of voxels showing higher mutual information in the attended 456 relative to the unattended condition was $55.1 \% \pm 0.9 \%$ (mean \pm SEM 457 across participants), which was significantly different from the chance 458 level of $50 \%(p<0.001, t(17)=5.81)$. Thus, attention reduced the un- 459 certainty of BOLD responses about object identity, implying enhanced 460 object coding at the level of single voxels.

\subsection{Attention enhances object representations at the pattern level}

A growing body of evidence suggests that the LOC codes object not 463 by means of individual neurons or neuronal populations, but across 464 multiple distributed neuronal populations (Haxby et al., 2001; Rice 465 et al., 2014). Thus, if attention has an enhancing effect on sensory repre- 466 sentations, the above finding of object-specific local modulation by at- 467 tention should improve the quality of multi-voxel activation patterns. 468

In a first step we assessed the effect of attention on the reproducibil- 469 ity of activation patterns by computing the within-object pattern simi- 470 larity (WPS) of activation patterns across repeated presentations of 471 the same object, separately for attended and unattended object presen- 472 tations. We found that attention significantly increased WPS ( $p<0.001,473$ $\mathrm{t}(17)=10.51$, two-tailed t-test; Fig. 4A), indicating that attention im- 474 proved the reproducibility of responses at the pattern level. However, 475 in a control analysis we found that attention also led to a considerable 476 increase of the between-object pattern similarity (BPS; $\mathrm{p}<0.001,477$ $\mathrm{t}(17)=9.73$, two-tailed $\mathrm{t}$-test). If the increase in reproducibility for 478 the same object (WPS) was outweighed by a simultaneous increase of 479 the ambiguity between different objects (BPS), nothing is gained. We 480 therefore directly compared WPS and BPS and found that the increase 481 in WPS was greater than the increase in BPS ( $p<0.001, \mathrm{t}(17)=4.22), 482$ indicating that attention led to a functionally relevant improvement of 483 the reproducibility of multi-voxel activation patterns.
A

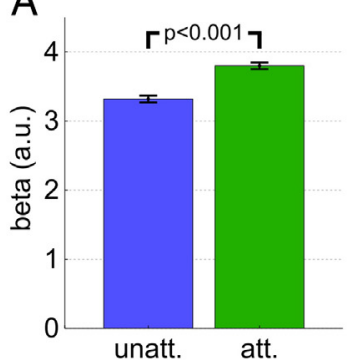

B

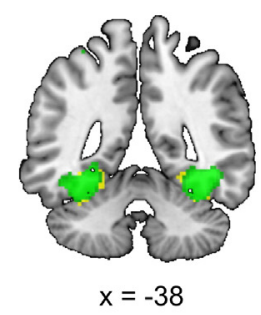

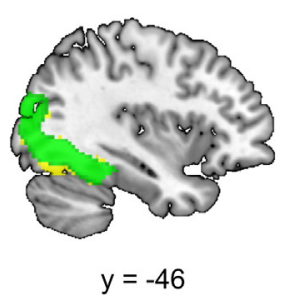

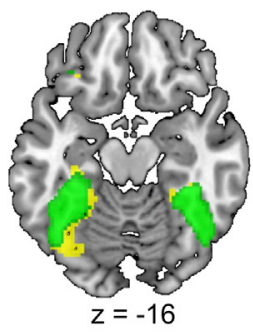

localizer attention

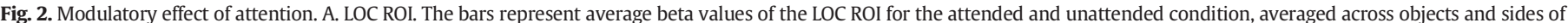

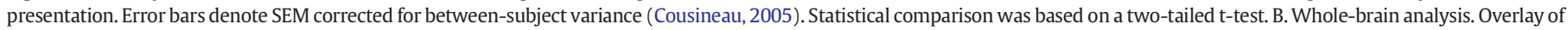

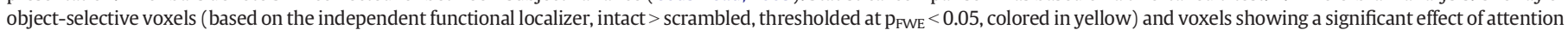
(attended $>$ unattended, thresholded at $\mathrm{p}_{\mathrm{FWE}}<0.05$, colored in green). 


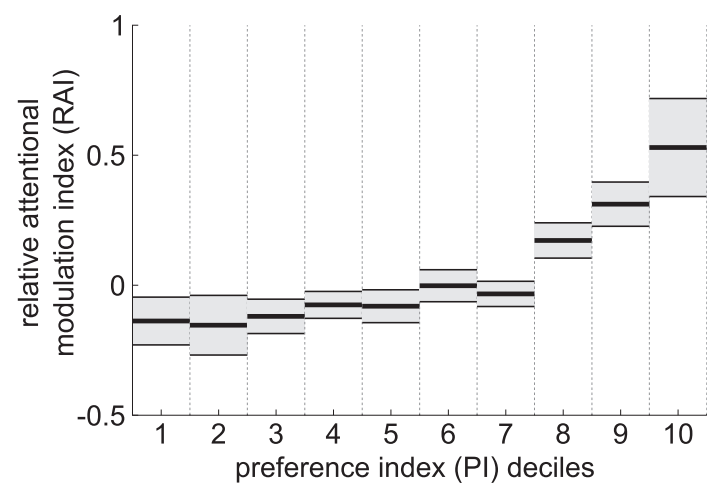

Fig. 3. Relative attentional modulation as a function of object preference. The relative attentional modulation index (RAI) quantifies the attentional modulation for a given object relative to the average modulation of the other objects. For each participant voxels were binned into deciles according to their object preference index (PI). The plot shows the averaged RAI for each preference bin. Error bars denote SEM corrected for between-subject variance (Cousineau, 2005)

In a second step we directly assessed how attention affected the readout of object information from the LOC by performing support vector machine classification between objects. Decoding accuracies were significantly above chance in both the attended $(66.6 \% \pm 2.0 \%$; $\mathrm{p}<0.001, \mathrm{t}(17)=8.36$, two-tailed t-test against the chance decoding accuracy of $50 \%)$ and the unattended condition $(54.8 \% \pm 1.8 \%$; $\mathrm{p}=$ $0.017, \mathrm{t}(17)=2.66$; Fig. 4B). Importantly, classification performance was significantly and markedly greater in the attended compared to the unattended condition $(\mathrm{t}(17)=4.74, \mathrm{p}<0.001$, Cohen's $\mathrm{d}=1.12$ ). Thus, the attentional modulation of neuronal responses increased object information in the LOC at the multi-voxel pattern level.

3.6. Enhanced readout at the pattern level is linked to the local increase in mutual information, but not mean activation

Finally, we assessed whether the attentional modulation at the single-voxel level was related to the enhancement of object representations at the pattern level. In the single-voxel-level analyses we found that attention led to an increase of (1) BOLD signal, and (2) the mutual information. We therefore correlated - across participants - both effects with the increase in decoding accuracy. We found that the increase in decoding accuracy correlated with the increase in mutual information (Pearson's $r=0.59, \mathrm{p}=0.009$ ), but not with the increase in BOLD activation $(r=0.01, p=0.96)$. A direct comparison confirmed that the

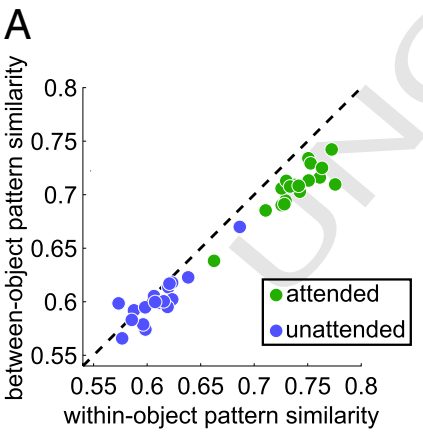

B

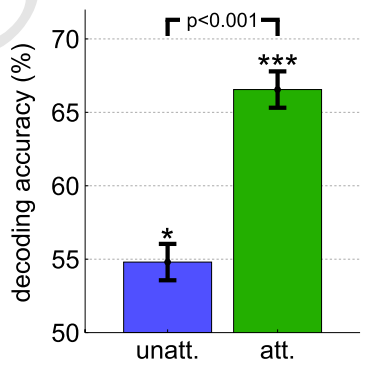

Fig. 4. Pattern level. A, between-object and within-object pattern similarity within the LOC ROI. Each dot represents one participant. The dashed diagonal line indicates identical within- and between-object similarity of activation patterns. Attention leads to a shift of data points below the diagonal line, indicating higher pattern similarity for repeated presentations of the same object compared to the pattern similarity of different objects. Between-subject variance was removed for illustration. B, SVM decoding results based on percent correct classification (decoding accuracy). Error bars denote SEM corrected for between-subject variance (Cousineau, 2005). Statistical comparison was based on a twotailed t-test. increase in mutual information explained significantly more variance 507 than the BOLD increase ( $\mathrm{p}=0.034, \mathrm{z}$-score $=2.11$, Steiger's z-test; 508 Steiger, 1980). Although the absence of a significant contribution of 509 the BOLD increase is surprising (possibly caused by ceiling effects of 510 the BOLD increase), the relationship between mutual information and 511 decoding accuracy suggests that the local attentional modulation of 512 neuronal responses increases the information content of object repre- 513 sentations at the pattern level.

\section{Discussion}

515

We examined tuning-dependent attentional modulation of object 516 representations in the LOC and the resulting enhancement of object rep- 517 resentations at the single-voxel level and the multi-voxel pattern level. 518 At the single-voxel level we found that (1) responses in the LOC were 519 considerably stronger when an object was attended relative to when a 520 noise stimulus was attended; (2) the relative attentional modulation 521 (the attentional modulation for a given object relative to the average 522 modulation of other objects) increased as a function of a voxel's prefer- 523 ence for the given object; and (3) mutual information between a voxel's 524 responses and object identity increased, demonstrating that responses 525 became more informative about a presented object when the object 526 was attended compared to when it was unattended. All three results 527 provide evidence against a mere baseline-shift effect of attention. Fur- 528 ther analyses showed that these local changes resulted in increased ob- 529 ject information at the level of multi-voxel patterns and increased 530 similarity of these patterns across multiple presentations, indicating in- 531 creased reproducibility of distributed neuronal responses.

532

\subsection{Effects of attention at the level of individual voxels}

A key goal of this study was to investigate whether the observed in- 534 crease in activity involved amplificatory attentional modulation, or 535 merely an unspecific baseline shift. Previous neuroimaging studies re- 536 ported that neural activity increased with attention in high-level visual 537 cortex (Baldauf and Desimone, 2014; Connor et al., 1997; Murray and 538 Wojciulik, 2004; O'Craven et al., 1999; Serences et al., 2004), and 539 showed that the effects of attention were specific to coarse functional 540 modules, such as parahippocampal place area (PPA) or fusiform face 541 area (FFA). However, given that objects are known to be coded across 542 distributed neuronal ensembles in visual cortex (Haxby et al., 2001; 543 Rice et al., 2014), it is desirable to analyze attentional modulation at a 544 more fine-grained level, thereby accounting for the differential tuning 545 of neuronal populations within these areas. Here we provide evidence 546 for voxel-wise object-specific attentional modulation of responses in 547 the LOC by identifying a relationship between attentional modulation 548 and object preference. The consistent increase of the relative attentional 549 modulation across preference levels suggests that subtle difference in 550 preference measured in the absence of attention became amplified as 551 attention was directed to the objects. Our additional information- 552 theoretic analyses indicated that such attentional modulation effective- 553 ly increased the information of voxel-wise responses about object iden- 554 tity, in line with previous work on orientation coding in V1, which 555 likewise found an increase in mutual information with attention 556 (Saproo and Serences, 2010).

557

How do these results relate to the multiplicative gain hypothesis of 558 attention derived from neurophysiological recordings in monkeys 559 (McAdams and Maunsell, 1999; Treue and Martínez Trujillo, 1999)? It 560 should be noted that a direct comparison between the BOLD responses 561 in our study and spiking activity in these previous studies is difficult for 562 two reasons: first, BOLD responses are more closely related to the local 563 field potentials and hence synaptic activity than to spiking neuronal ac- 564 tivity (Ekstrom, 2010; Logothetis, 2003; Logothetis et al., 2001); and 565 second, efficient event-related fMRI designs such as ours do not permit 566 inferences about the absolute level of stimulus-related BOLD activity, 567 which would be necessary to quantify the ratio between attended and 568 
unattended responses analogous to the ratio of firing rates in these previous neurophysiological studies. Nevertheless, our results do provide indirect evidence for the multiplicative gain as opposed to a mere baseline shift hypothesis. Consider the result of increased attentional modulation with object preference. A voxel's preference for a given object may indicate that, for a fixed number of neurons tuned to different objects, the tuning curves of neurons are biased more towards the given object than to the other objects. Alternatively, it may indicate that for a fixed bias towards the given object an overall greater number of neurons prefer the given object. Importantly, in both cases an unspecific baseline shift would lead to an equal increase of neural activity for preferred and non-preferred objects, which is at odds with our results. To illustrate why the increase in MI provides evidence for a multiplicative gain mechanism as opposed to a pure baseline shift explanation, it is helpful to consider two objects A and B and a hypothetical voxel consisting of neurons with a preference for, e.g., object A. In case of a pure baseline shift the voxel would show increased responses to both objects and neural responses would therefore not become more informative about whether object A or B was presented. In contrast, in case of multiplicative scaling, attention will lead to greater response amplification for object A compared to object $\mathrm{B}$, increasing the dynamic range of responses and resulting in increased mutual information between neural responses and presented objects. Thus, the increase in mutual information by attention provides a second line of evidence in favor of a multiplicative gain mechanism and against a pure baseline shift explanation.

\subsection{Effects of attention at the multi-voxel pattern level}

At the level of multi-voxel activation patterns we found improved decodability of attended relative to unattended objects, which is in accordance with similar reports for early (Jehee et al., 2011; Kamitani and Tong, 2005) and high-level visual areas (Pratte et al., 2013; Reddy and Kanwisher, 2007). This result demonstrates that the attentional modulation increased the information content of distributed object representations in the LOC, potentially benefitting information readout from the LOC by high-level executive cortices. An analysis of pattern similarity showed that attention increased the reproducibility of activation patterns of the same object. Such an increase in reproducibility would be expected on the assumption of a multiplicative attentional scaling mechanism, where neuronal responses become amplified without an equivalent increase of the noise (which increases as the squareroot of the signal). Another possibility is that the increase in reproducibility is the result of more discrete neural processing with attention, as proposed for conscious relative to non-conscious percepts (Sackur and Dehaene, 2009; Schurger et al., 2010). When discrete decisions are reached at each (object) processing stage, before they are dispatched to the next stage, the resulting activation patterns might become more stereotypical and reproducible.

A number of previous fMRI studies have used MVPA to study the effects of attention on neural responses (Esterman et al., 2009; Jiang et al., 2013; Pratte et al., 2013; Reddy and Kanwisher, 2007; Reddy et al., 2009; Tamber-Rosenau et al., 2011). In particular, Reddy and Kanwisher (2007) and Reddy et al. (2009) investigated the decodability of complex stimuli in high-level visual cortex when they were presented alongside a second object and were either attended or unattended. Reddy and Kanwisher (2007) found that information about object categories encoded in multi-voxel activation patterns was strongly reduced to the point of being abolished when attention was diverted. In the present study we showed that multi-voxel responses were reduced, but still informative about object categories even when attention was diverted. This difference may be explained by the fact that participants in the study by Reddy and Kanwisher (2007) directed their attention to complex distractor stimuli (which, in addition, were the relevant stimuli in other trials), whereas participants in our study viewed noise stimuli in the unattended condition. It is conceivable that the absence of high- level visual cortex information for unattended objects in Reddy and 633 Kanwisher (2007) was caused by distractor-related neural responses 634 interfering with the activation pattern of the unattended target object. 635 Along similar lines, Reddy et al. (2009) interpreted the informational 636 gain for attended objects (or loss for unattended objects) in the biased 637 competition framework. According to this view, attention serves to dis- 638 ambiguate the overlapping multi-voxel patterns of different objects 639 through a shift towards the pattern of the currently attended object. 640 Aside from investigating the effect of attention in sensory cortices, 641 other studies have successfully used MVPA to study the initiation and 642 control of attentional shifts. For instance, Esterman et al. (2009) and 643 Tamber-Rosenau et al. (2011) showed that spatial patterns of brain ac- 644 tivity within the medial superior parietal lobule reliably differentiated 645 between several domains of cognitive attentional control at a given mo- 646 ment. Thus, in our and previous studies, MVPA presented a powerful 647 technique to probe distributed neural underpinnings of different atten- 648 tional phenomena, from the initiation of attentional shifts to the modu- 649 lation of sensory representations.

\subsection{Linking the single-voxel and the multi-voxel pattern level}

Finally, we linked the effects of attention at the single-voxel level 652 with the effects at the pattern levels by correlating the increase in 653 decoding accuracy of multi-voxel activation patterns to the increase in 654 either BOLD signal or mutual information. Unexpectedly, we found 655 that the increase in mean activation was not related to the increase in 656 decoding accuracy. This negative finding could indicate that the atten- 657 tional manipulation in our paradigm operated in a range, in which ef- 658 fects at the pattern level were insensitive to the overall magnitude 659 (e.g., because the BOLD increase was at maximum). Alternatively, as 660 the overall effect of attention likely involves both a multiplicative com- 661 ponent and a baseline shift, the unspecific baseline shift component 662 might have masked the effect of the relevant multiplicative component. 663 In contrast, we found that the increase in mutual information explained 664 a considerable amount of variance of improvements in pattern-based 665 decoding. This result demonstrates that the increase of object informa- 666 tion at the single-voxel level substantially translated to an enhanced ob- 667 ject code at the pattern level. This link is informative, as the information 668 content encoded in the linear combination of voxels can show strong 669 gains, while information encoded in the individual voxel may show 670 only small changes (for examples of such scenarios see Haynes and 671 Rees, 2006). It is currently not clear whether the distributed object 672 code in LOC represents the immediate neural correlate of perception, 673 or whether it reflects object processing prior to perception. In either 674 case our data indicate that the enhancement of sensory representations 675 through attention - which may directly or indirectly underlie perceptu- 676 al improvements - is not a phenomenon that solely emerges at the level 677 of distributed object fingerprints. Instead, the improvement in pattern 678 decoding likely represents the cumulative result of informational gains 679 in multiple local units of LOC.

\subsection{Implications for mechanisms of visual attention}

681

The results of the present study corroborate the notion that behav- 682 ioral benefits of attention are based on an enhanced stimulus processing 683 in sensory brain areas (Bisley, 2011). Our finding that the magnitude of 684 attentional modulation increased with object preference suggests a re- 685 sponse gain mechanism that magnifies stimulus-driven responses as a 686 function of response strength without attention. Importantly, our 687 information-theoretic analyses demonstrate that the attentional modu- 688 lation effectively increases object information encoded in high-level vi- 689 sual cortex, which may facilitate the readout in executive cortices and 690 thus benefit perceptual decision making. A unifying theoretical frame- 691 work for such attentional modulation of neural activity is provided by 692 the normalization model of attention (Reynolds and Heeger, 2009). 693 The model describes the modulation of attention by two processes: a 694 
multiplication of neuronal responses by an attention field and a division (normalization) by a suppressive drive. Thus, our observed differences between neural responses to attended and unattended objects may not only be caused by a boost of neural processes tuned to the attended object, but also by a suppression of activity related to the unattended object. Another key aspect of the model is that it makes specific predictions regarding the effect of different attentional strategies on neural activity. According to the model, a purely spatial attention strategy causes a scaling of the entire tuning curves (because the attention field is then assumed to be constant across feature dimensions), whereas a purely feature-based attention strategy causes a sharpening of tuning curves. The fact that our brightness discrimination task emphasized spatial attention strategies over feature-based strategies may thus explain the strong amplitude modulation of the BOLD response in our study. Future neuroimaging studies could test whether our findings of tuningdependent attentional modulation and information-theoretic gains through endogenous visual spatial attention generalize to other forms of attention, e.g. to involuntary (exogenous) shifts of attention or to other sensory modalities.

In conclusion, our results show that visual spatial attention modulates neural activity as a function of voxel-based object preferences. Through these modulatory processes, attention enhances object coding both at the single-voxel and pattern level, which may give rise to improved perception and perceptual decisions.

\section{Acknowledgments}

This research was supported by the German Research Foundation (DFG) through the Research Training Group GRK1589/1 (to M.G. and P.S.), and Grants STE1430/6-1 (to P.S.), and RI1847/1-1 and SFB779TPA10N (to A.R.-K). R.C. was funded by a Feodor Lynen Grant of the Alexander von Humboldt Foundation. We thank Guy Middleton for assistance with rendering the object images from 3D models.

\section{References}

Avidan, G., Harel, M., Hendler, T., Ben-Bashat, D., Zohary, E., Malach, R., 2002. Contrast sensitivity in human visual areas and its relationship to object recognition. J. Neurophysiol. 87 3102-3116.

Baldauf, D., Desimone, R., 2014. Neural mechanisms of object-based attention. Science 344 , 424-428.

Bashinski, H.S., Bacharach, V.R., 1980. Enhancement of perceptual sensitivity as the result of selectively attending to spatial locations. Percept. Psychophys. 28, 241-248.

Bisley, J.W., 2011. The neural basis of visual attention. J. Physiol. 589, 49-57.

Cameron, E.L., Tai, J.C., Carrasco, M., 2002. Covert attention affects the psychometric function of contrast sensitivity. Vis. Res. 42, 949-967.

Carrasco, M., Penpeci-Talgar, C., Eckstein, M., 2000. Spatial covert attention increases contrast sensitivity across the CSF: support for signal enhancement. Vis. Res. 40, 1203-1215.

Connor, C.E., Preddie, D.C., Gallant, J.L., Essen, D.C. Van, 1997. Spatial attention effects in macaque area V4. J. Neurosci. 17, 3201-3214.

Cousineau, D., 2005. Confidence intervals in within-subject designs: a simpler solution to Loftus and Masson's method. Tutor. Quant. Methods Psychol. 1, 42-45.

Di Russo, F., Spinelli, D., Morrone, M.C., 2001. Automatic gain control contrast mechanisms are modulated by attention in humans: evidence from visual evoked potentials. Vis. Res. 41, 2435-2447.

Ekstrom, A., 2010. How and when the fMRI BOLD signal relates to underlying neural activity: the danger in dissociation. Brain Res. Rev. 62, 233-244.

Esterman, M., Chiu, Y., Tamber-rosenau, B.J., Yantis, S., 2009. Decoding cognitive control in human parietal cortex. Proc. Natl. Acad. Sci. U. S. A. 106, 17974-17979.

Gandhi, S.P., Heeger, D.J., Boynton, G.M., 1999. Spatial attention affects brain activity in human primary. Proc. Natl. Acad. Sci. U. S. A. 96, 3314-3319.

Gitelman, D.R., Nobre, A.C., Parrish, T.B., LaBar, K.S., Kim, Y.H., Meyer, J.R., Mesulam, M., 1999. A large-scale distributed network for covert spatial attention: further anatomical delineation based on stringent behavioural and cognitive controls. Brain 122, 1093-1106.

Guggenmos, M., Thoma, V., Cichy, R.M., Haynes, J.-D., Sterzer, P., Richardson-Klavehn, A., 2015. Non-holistic coding of objects in lateral occipital complex with and without attention. Neuroimage 107, 356-363.

Hawkins, H.L., Hillyard, S.A., Luck, S.J., Mouloua, M., Downing, C.J., Woodward, D.P., 1990. Visual attention modulates signal detectability. J. Exp. Psychol. Hum. Percept. Perform. 16, $802-811$.

Haxby, J.V., Gobbini, M.I., Furey, M.L., Ishai, A., Schouten, J.L., Pietrini, P., 2001. Distributed and overlapping representations of faces and objects in ventral temporal cortex. Science 293, 2425-2430.

Haynes, J.-D., Rees, G., 2005. Predicting the orientation of invisible stimuli from activity in human primary visual cortex. Nat. Neurosci. 8, 686-691.
Haynes, J.-D., Rees, G., 2006. Decoding mental states from brain activity in humans. Nat. Rev. 766 Neurosci. 7, 523-5

Hebart, M.N., Görgen, K., Haynes, J.-D., 2014. The Decoding Toolbox (TDT): a versatile software 768 package for multivariate analyses of functional imaging data. Front. Neuroinform. 8.

Jehee, J.F.M., Brady, D.K., Tong, F., 2011. Attention improves encoding of task-relevant features in 770 the human visual cortex. J. Neurosci. 31, 8210-8219.

Jiang, J., Summerfield, C., Egner, T., 2013. Attention sharpens the distinction between expected 772 and unexpected percepts in the visual brain. J. Neurosci. 33, 18438-18447.

Kamitani, Y., Tong, F., 2005. Decoding the visual and subjective contents of the human brain. 774 Nat. Neurosci. 8, 679-685.

Kastner, S., Pinsk, M.A., De Weerd, P., Desimone, R., Ungerleider, L.G., 1999. Increased activity in 776 human visual cortex during directed attention in the absence of visual stimulation. Neuron 777 22, 751-761

Kojadinovic, I., 2005. On the use of mutual information in data analysis: an overview. Proceed- 779 ings of the 11th International Symposium on Applied Stochastic Models and Data Analysis 780 (ASMDA '05), pp. 738-747.

Logothetis, N.K., 2003. The underpinnings of the BOLD functional magnetic resonance imaging 782 signal. J. Neurosci. 23, 3963-3971.

Logothetis, N.K. Pauls, J., Augath, M., Trinath, T., Oeltermann, A., 2001. Neurophysiological inves- 784 tigation of the basis of the fMRI signal. Nature 412, 150-157.

Luck, S.J., Chelazzi, L., Hillyard, S.A., Desimone, R., 1997. Neural mechanisms of spatial selective 786 attention in areas V1, V2, and V4 of macaque visual cortex. J. Neurophysiol. 77, 24-42. 787

Martínez, A., Anllo-Vento, L., Sereno, M.I., Frank, L.R., Buxton, R.B., Dubowitz, D.J., Wong, E.C., 788 Hinrichs, H., Heinze, H.J., Hillyard, S.A., 1999. Involvement of striate and extrastriate visual 789 cortical areas in spatial attention. Nat. Neurosci. 2, 364-369. 790

McAdams, C.J., Maunsell, J.H.R., 1999. Effects of attention on orientation-tuning functions of sin- 791

$\begin{array}{cc}\text { gle neurons in macaque cortical area V4. J. Neurosci. 19, 431-441. } & 792 \\ \text { Motter, B.C., 1993. Focal attention produces spatially selective processing in visual cortical areas } & 793\end{array}$ V1, V2, and V4 in the presence of competing stimuli. J. Neurophysiol. 70, 909-919. 794

Mumford, J.A., Davis, T., Poldrack, R.A., 2014. The impact of study design on pattern estimation 795 for single-trial multivariate pattern analysis. Neuroimage 103, 130-138. 796

Murray, S.O., He, S., 2006. Contrast invariance in the human lateral occipital complex depends 797 on attention. Curr Biol. 16, 606-611.

Murray, S.O., Wojciulik, E., 2004. Attention increases neural selectivity in the human lateral oc- 799 cipital complex. Nat. Neurosci. 7, 70-74.

O'Connor, D.H., Fukui, M.M., Pinsk, M.A., Kastner, S., 2002. Attention modulates responses in the 801 human lateral geniculate nucleus. Nat. Neurosci. 5, 1203-1209. 802

O'Craven, K.M. Downing P.E, Kanwisher, N., 1999. FMRI evidence for objects as the units of at- 803 tentional selection. Nature 401, 584-587.

Pratte, M.S., Ling, S., Swisher, J.D., Tong, F., 2013. How attention extracts objects from noise. 805 J. Neurophysiol. 110, 1346-1356.

Reddy, L., Kanwisher, N., 2007. Category selectivity in the ventral visual pathway confers robust- 807 ness to clutter and diverted attention. Curr. Biol. 17, 2067-2072

Reddy, L., Kanwisher, N.G., VanRullen, R., 2009. Attention and biased competition in multi-voxel 809 object representations. Proc. Natl. Acad. Sci. U. S. A. 106, 21447-21452. 810

Ress, D., Backus, B.T., Heeger, D.J., 2000. Activity in primary visual cortex predicts performance in 811 a visual detection task. Nat. Neurosci. 3, 940-945. 812

Reynolds, J.H., Heeger, D.J., 2009. The normalization model of attention. Neuron 61, 168-185. 813

Reynolds, J.H., Pasternak, T., Desimone, R., 2000. Attention increases sensitivity of V4 neurons. 814 Neuron 26, 703-714.

Rice, G.E., Watson, D.M., Hartley, T., Andrews, T.J., 2014. Low-level image properties of visual ob- 816 jects predict patterns of neural response across category-selective regions of the ventral vi- 817 sual pathway. J. Neurosci. 34, 8837-8844.

Rolls, E.T., Baylis, G.C., 1986. Size and contrast have only small effects on the responses to faces of 819 neurons in the cortex of the superior temporal sulcus of the monkey. Exp. Brain Res. 65, 820 38-48. 821

Sackur, J., Dehaene, S., 2009. The cognitive architecture for chaining of two mental operations. 822 Cognition 111, 187-211. 823

Saproo, S., Serences, J.T., 2010. Spatial attention improves the quality of population codes in 824 human visual cortex. J. Neurophysiol. 104, 885-895. 825

Saproo, S., Serences, J.T., 2014. Attention improves transfer of motion information between V1 826 and MT. J. Neurosci. 34, 3586-3596.

Schurger, A., Pereira, F., Treisman, A., Cohen, J.D., 2010. Reproducibility distinguishes conscious 828 from nonconscious neural representations. Science 327, 97-99. 829

Serences, J.T., Schwarzbach, J., Courtney, S.M., Golay, X., Yantis, S., 2004. Control of object-based 830 attention in human cortex. Cereb. Cortex 14, 1346-1357.

Serences, J.T., Saproo, S., Scolari, M., Ho, T., Muftuler, L.T., 2009. Estimating the influence of atten- 832 tion on population codes in human visual cortex using voxel-based tuning functions. 833 Neuroimage 44, 223-231.

Silver, M.A., Ress, D., Heeger, D.J., 2007. Neural correlates of sustained spatial attention in human 835 early visual cortex. J. Neurophysiol. 97, 229-237. (19., Dunctional MRI reveals spatially spe- 837 cific attentional modulation in human primary visual cortex. Proc. Natl. Acad. Sci. U. S. A. 96, 838 1663-1668.

Steiger, J.H., 1980. Test for comparing elements of a correlation matrix. Psychol. Bull. 87, 840 245-251.

Tamber-Rosenau, B.J., Esterman, M., Chiu, Y.-C., Yantis, S., 2011. Cortical mechanisms of cogni- 842 tive control for shifting attention in vision and working memory. J. Cogn. Neurosci. 23, 843 2905-2919. 844

Treue, S., 2003. Visual attention: the where, what, how and why of saliency. Curr. Opin. 845 Neurobiol. 13, 428-432.

Treue, S., Martínez Trujillo, J.C., 1999. Feature-based attention influences motion processing gain 847 in macaque visual cortex. Nature 399, 575-579.

Treue, S., Maunsell, J.H.R., 1999. Effects of attention on the processing of motion in macaque 849 middle temporal and medial superior temporal visual cortical areas. J. Neurosci. 19, 850 7591-7602.

Williford, T., Maunsell, J.H.R., 2006. Effects of spatial attention on contrast response functions in 852 macaque area V4. J. Neurophysiol. 96, 40-54. 